\title{
Nonlinear System Identification Using Exponential Swept-Sine Signal
}

\author{
Antonin Novak ${ }^{1} *$, Laurent Simon ${ }^{1}$, Frantisek Kadlec ${ }^{2}$, Pierrick Lotton ${ }^{1}$ \\ ${ }^{1}$ Laboratoire d'Acoustique de I'Université du Mans, LAUM - UMR 6613 CNRS, Le Mans Université, Avenue Olivier \\ Messiaen, 72085 LE MANS CEDEX 9, France \\ ${ }^{2}$ Czech Technical University in Prague, Faculty of Electrical Engineering, Prague, Czech Republic \\ *antonin.novak@univ-lemans.fr, ${ }^{*}$ https://ant-novak.com
}

\begin{abstract}
In this paper, we propose a method for nonlinear system (NLS) identification using a swept-sine input signal and based on nonlinear convolution. The method uses a nonlinear model, the non-parametric generalized polynomial Hammerstein model made of power series associated with linear filters. Simulation results show that the method identifies the nonlinear model of the system under test and estimates the linear filters of the unknown NLS. The method has been also tested on a real-world system: an audio limiter. Once the nonlinear model of the limiter is identified, a test signal can be regenerated to compare the outputs of both the real-world system and its nonlinear model. The results show good agreement between both model-based and real-world system outputs.
\end{abstract}

The archived file is not the final published version of the article A. Novak, L. Simon, F. Kadlec \& P. Lotton (2010),

"Nonlinear system identification using exponential swept-sine signal", Instrumentation and Measurement, IEEE

Transactions on. Vol. 59(8), pp. 2220-2229.

The definitive publisher-authenticated version is available online at https://doi.org/10.1109/TIM.2009.2031836, Readers must contact the publisher for reprint or permission to use the material in any form. 


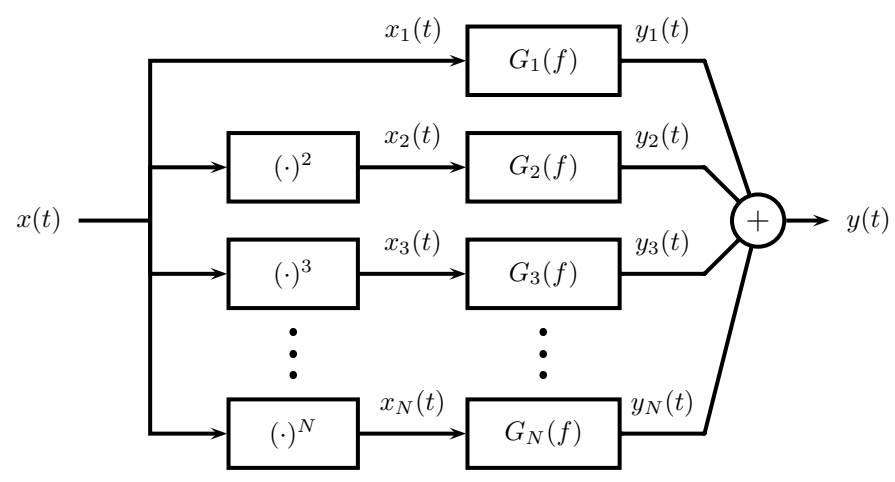

Figure 1. Generalized Polynomial Hammerstein model (power series nonlinear model).

\section{Introduction}

The theory of linear time-invariant (LTI) systems has been extensively studied over decades [1, 2] and the estimation of any unknown LTI system, knowing both the input and output of the system, is a solved problem. The fundamental idea of the theory states that any LTI system can be characterized entirely by its impulse response in the time domain or by its frequency response function in the frequency domain. Nevertheless, almost all real-world devices exhibit more or less nonlinear behavior. In the case of very weak nonlinearities, a linear approximation can be used. If the nonlinearities are stronger, the linear approximation fails and the system has to be described using a nonlinear model. Such nonlinear models are available in the literature: for example, Volterra model [3], neural network model [4], MISO model [5], NARMAX model [6, 7], hybrid genetic algorithm [8], extended Kalman filtering [9], particle filtering [10].

All these models involve parameters or kernels that have to be estimated. If a theoretical physical model of the nonlinear system (NLS) under test is available, the global nonlinear behavior of the system is known and the method to be carried out consists in the estimation of the unknown parameters of the NLS. If no prior knowledge of the NLS is available, an identification procedure has to be involved. This procedure is based on the analysis of the signal produced at the output of the system under test when exciting the system by a given and controlled input signal. Different input signals can be used, depending on the method chosen for the estimation, such as sine wave excitation, multitone excitation [11, 12, 13], random noise excitation [5], pseudorandom signals [14, 15].

Regarding the basics of NLS theory, when a pure sine-wave input signal $x(t)=A_{1} \cos \left(2 \pi f_{1} t+\phi_{1}\right)$ passes through a NLS, higher-order harmonic components appear at the output of the system as multiples of input frequency, according to $y(t)=\sum_{n} B_{n} \cos \left(2 \pi n f_{1} t+\phi_{n}\right)$. The characteristics (amplitude $B_{n}$ and phase $\phi_{n}$ ) of all higher-order components may be furthermore frequency and input amplitude dependent, in the sense that $\forall n B_{n} \equiv B_{n}\left(A_{1}, f_{1}\right)$, $\phi_{n} \equiv \phi_{n}\left(A_{1}, f_{1}\right)$. The complete identification procedure consequently needs to estimate the amplitude $B_{n}$ and phase $\phi_{n}$ as functions of input amplitude and frequency. Nonlinear models including frequency dependency are for example Wiener model, Hammerstein model, or Wiener-Hammerstein model [15].

A non-parametric generalized polynomial Hammerstein model [15] of order $N$ is considered here, as illustrated in Fig. 1. The model is made up of $N$ parallel branches, each branch consisting of a linear filter $G_{n}(f)$ preceded by a $n$-th power static nonlinear function. Giving both the input and output signals $x(t)$ and $y(t)$, the problem of identifying the generalized polynomial Hammerstein model consists then in the estimation of the unknown linear filters $G_{n}(f), n \in[1, N]$. 


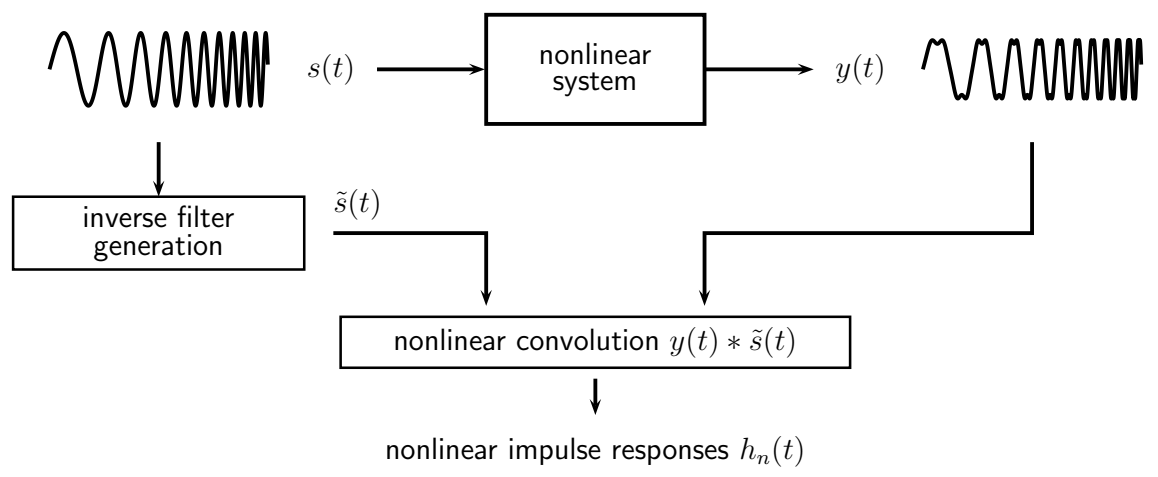

Figure 2. Block diagram of the nonlinear convolution process in NLS identification.

In this paper, we propose a new method for identifying the generalized polynomial Hammerstein model based on an input exponential swept-sine signal, allowing a one-path estimation of the unknown linear filters. The paper is organized as follows. In Sect. 2, we review the basics of the nonlinear convolution firstly proposed in $[16,17]$ and we recall the properties of asymptotic signals. In Sect. 3, we define the swept-sine input signal and we detail how to design this input signal for the purpose of NLS identification. In Sect. 4, we define the associated inverse filter and we show how to estimate the linear filters of the model. In Sect. 5, we show the results of numerical experiments and real NLS identification (audio limiter). In Sect. 6, the proposed method is compared with other methods based on dedicated models [18], such as Hammerstein or Wiener models. Finally, the conclusions are noted in Sect. 7.

\section{Basics of the method}

The method presented in this paper is partly based on nonlinear convolution method presented in [17]. The method uses a swept-sine signal (also called a chirp), exhibiting an exponential instantaneous frequency, as the excitation signal and allows the characterization of a NLS in terms of harmonic distortion at several orders. This nonlinear convolution method and the basic theory of instantaneous frequency and complex signal are reviewed in this section.

\subsection{Nonlinear Convolution}

The block diagram of the method is shown in Fig. 2. First, an exponential swept-sine signal $s(t)$ is generated and used as the input signal of the nonlinear system under test. The distorted output signal $y(t)$ is recorded for being used for the so-called nonlinear convolution [17]. Next, the signal noted $\tilde{s}(t)$ is derived from the input signal $s(t)$ as its time-reversed replica with amplitude modulation such that the convolution between $s(t)$ and $\tilde{s}(t)$ gives a Dirac delta function $\delta(t)$. The signal $\tilde{s}(t)$ is called "inverse filter" [16, 17]. Then, the convolution between the output signal $y(t)$ and the inverse filter $\tilde{s}(t)$ is performed. The result of this convolution can be expressed as

$$
y(t) * \tilde{s}(t)=\sum_{m=1}^{\infty} h_{m}\left(t+\Delta t_{m}\right)
$$

where $h_{m}(t)$ are higher-order impulse responses and $\Delta t_{m}$ are the time lag between the first (linear) and the $m$-th impulse response. Since the nonlinear impulse response consists of a set of higher-order impulse responses that are time shifted, each partial impulse response can be separated from each other, as illustrated by Fig. 3. This procedure is developed in [16]. 


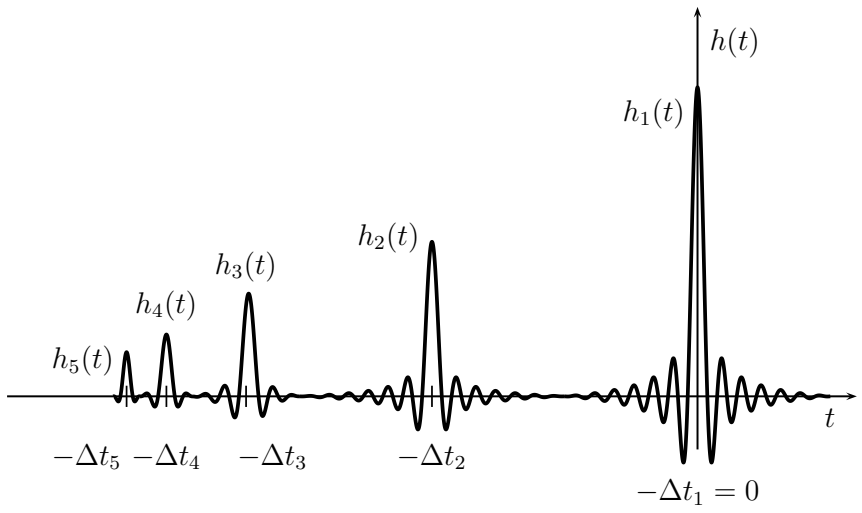

Figure 3. Result of the nonlinear convolution process $y(t) * \tilde{s}(t)$ in the form of set of higher-order nonlinear impulse responses $h_{m}(t)$.

The set of higher-order nonlinear impulse responses $h_{m}(t)$ can be also expressed in the frequency domain. The frequency response functions of higher-order nonlinear impulse responses $h_{m}(t)$ is then their Fourier transforms

$$
H_{m}(f)=\mathbf{F T}\left[h_{m}(t)\right]
$$

The frequency responses $H_{m}(f)$ represent the frequency dependency of higher-order components. The frequency response $H_{1}(f)$ is consequently the response corresponding to the linear part of the system. Similarly, the frequency response $H_{m}(f)(m>1)$ may be regarded as the system frequency response, when considering only the effect of the input frequency $f$ on the $m$-th harmonic frequency $m f$ of the output. The relation between partial frequency responses $H_{m}(f)$ and linear filters $G_{n}(f)$ is detailed in section 4 .

\subsection{Instantaneous frequency and complex signal}

A swept-sine signal, or chirp, is a signal in which the instantaneous frequency increases with time and can be generally defined as

$$
s(t)=a(t) \sin (\varphi(t)) .
$$

The analytic signal of the signal $s(t)$ is

$$
z_{s}(t)=s(t)+j H[s(t)]=a_{s}(t) e^{j \varphi_{s}(t)},
$$

where $H[]$ is the Hilbert transform and where $a_{s}(t)$ and $\varphi_{s}(t)$ are unambiguously defined as amplitude and phase of $z_{s}(t)$. The spectrum $Z_{s}(f)$ of the signal $z_{s}(t)$ can be written in terms of amplitude and phase as

$$
Z_{s}(f)=B_{s}(f) e^{j \Psi_{s}(f)} .
$$

The instantaneous frequency $f_{i}(t)$ and the group delay $t_{f}(t)$ are then defined as

$$
\begin{aligned}
f_{i}(t) & =\frac{1}{2 \pi} \frac{d \varphi_{s}(t)}{d t}, \\
t_{f}(f) & =-\frac{1}{2 \pi} \frac{d \Psi_{s}(f)}{d f} .
\end{aligned}
$$

Eq. (6) and (7) define two curves in the time-frequency planes which, for strictly monotonic chirps or so-called asymptotic signals $[19,20]$, may be regarded as inverse of each other. In such conditions of asymptotic signal, if one 
of these functions is known, the other one can then be easily calculated. Furthermore, we can write the following equalities $t \equiv t_{f} \equiv t_{f}(f)$ and $f \equiv f_{i} \equiv f_{i}(t)$.

These properties allow to know the spectra of the signal $z_{s}(t)$ with no need to calculate its Fourier transform. The amplitude $a_{s}(t)$ and the phase $\varphi_{s}(t)$ in time domain are related to the amplitude $B_{s}(f)$ and the phase $\Psi_{s}(f)$ in frequency domain, for $f>0$, as [21]

$$
\begin{aligned}
B_{s}(f) & =\frac{a_{s}\left(t_{f}\right)}{\sqrt{\frac{1}{2 \pi}\left|\varphi_{s}^{\prime \prime}\left(t_{f}\right)\right|}}, \\
\Psi_{s}(f) & =\varphi_{s}\left(t_{f}\right)-2 \pi f t_{f}+\frac{\pi}{4} \operatorname{sign}\left(\frac{d f_{i}\left(t_{f}\right)}{d t_{f}}\right) .
\end{aligned}
$$

\section{Input Signal and Inverse Filter: Design and properties}

In this section, we detail how to design the input signal used for the identification of the NLS under test. First, the input signal is defined as an exponential swept-sine signal as used in $[16,17]$. Then, a redesign of this input signal is proposed for the specific purpose of estimation of the linear filters of the non-parametric generalized polynomial Hammerstein model. Both time-domain and frequency-domain properties of this redesigned signal are also examined.

\subsection{Input Signal Design}

The input signal used for the identification is an exponential swept-sine signal, i.e. a signal exhibiting an instantaneous frequency which increases exponentially with time. Such a signal is also called an exponential chirp and is defined as

$$
\begin{aligned}
s(t) & =\sin \left[2 \pi f_{1} \int_{0}^{t} \exp \left(\frac{t^{\prime}}{L}\right) d t^{\prime}\right] \\
& =\sin \left\{2 \pi f_{1} L\left[\exp \left(\frac{t}{L}\right)-1\right]\right\},
\end{aligned}
$$

where $f_{1}$ is the start frequency at $t=0$, and $L$ is the rate of exponential increase in frequency. The parameter $L$ depends on the time length $T$ and the stop frequency $f_{2}$ of the swept-sine signal. The signal $s(t)$ defined in Eq. (10) can be also expressed as in Eq. (3) with constant amplitude envelope, $a(t)=1$, and the instantaneous phase

$$
\varphi(t)=2 \pi f_{1} L\left[\exp \left(\frac{t}{L}\right)-1\right]
$$

The instantaneous frequency, defined in (6), is

$$
f_{i}(t)=f_{1} \exp \left(\frac{t}{L}\right)
$$

The group delay $t_{f}$ is then the inverse function of instantaneous frequency $f_{i}$ and is given by

$$
t_{f}(f)=L \log \left(\frac{f_{i}}{f_{1}}\right) .
$$

The time length $T$ of the signal $s(t)$ can then be consequently expressed as the time between two particular instantaneous frequencies $f_{1}$ (start frequency) and $f_{2}$ (stop frequency),

$$
T=L \log \left(\frac{f_{2}}{f_{1}}\right),
$$




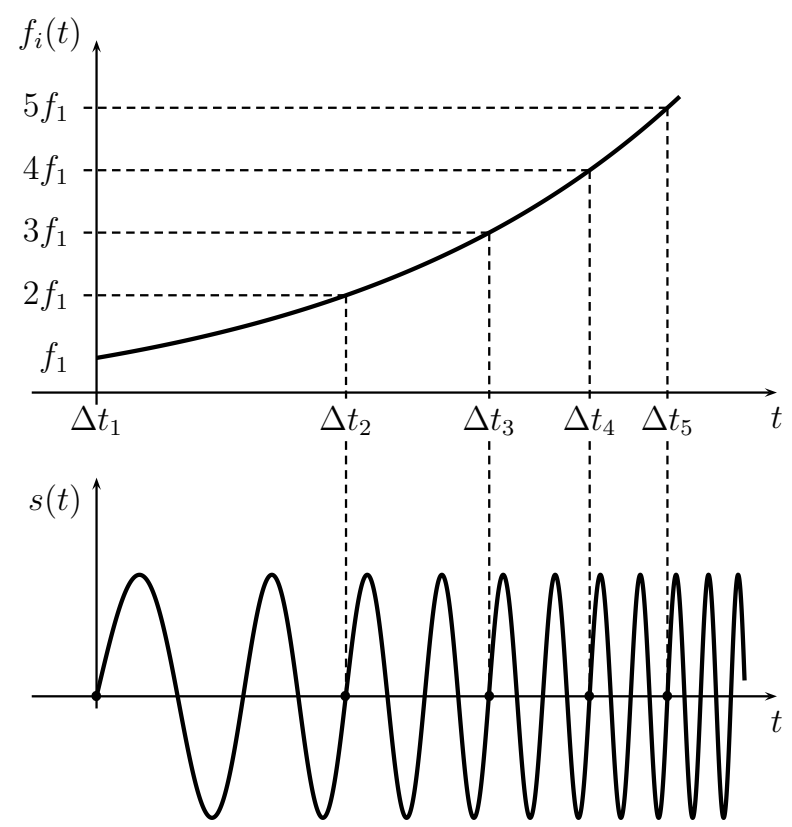

Figure 4. Swept-sine signal $s(t)$ in time domain (below), with the time length chosen according to instantaneous frequency $f_{i}(t)$ (above).

and thus the coefficient $L$ is defined as

$$
L=\frac{T}{\log \left(\frac{f_{2}}{f_{1}}\right)} .
$$

This definition of the swept-sine signal parameters is similar to the one used in $[16,17,22]$ for analysis of audio equipment nonlinearities. Nevertheless, for the nonlinear model estimation, this definition may lead to problems due to non-synchronization of the phases of the higher-order harmonic components. For that reason, the following procedure is proposed to redesign the exponential swept-sine signal.

Firstly, let $\Delta t_{m}$ be the time lag, for which the instantaneous frequency $f_{i}\left(\Delta t_{m}\right)$ is given by

$$
f_{i}\left(\Delta t_{m}\right)=m f_{1}
$$

for $m \in \mathbb{N}-\{0\}$. Using Eq. (13), it is then possible to write

$$
\Delta t_{m}=L \log (m),
$$

and the instantaneous phase at the time lag $\Delta t_{m}$ is given by

$$
\varphi\left(\Delta t_{m}\right)=2 \pi f_{1} L(m-1) .
$$

Secondly, as depicted in Fig. 4, the swept-sine signal $s(t)$ at particular time lag $\Delta t_{m}$ is designed to be equal to zero, $s\left(\Delta t_{m}\right)=0$, with the additional constraint of positive first derivative $s^{\prime}\left(\Delta t_{m}\right)>0$. These conditions consequently yields to

$$
\varphi\left(\Delta t_{m}\right)=2 k \pi
$$

where $k \in \mathbb{Z}$. Thus, from Eq. (19) and (18), we get

$$
f_{1} L(m-1)=k .
$$


Sufficient condition to solve (20) is then

$$
f_{1} L \in \mathbb{Z}
$$

Using this condition and Eq. (15), we write

$$
L=\frac{1}{f_{1}} \text { Round }\left(\frac{\hat{T} f_{1}}{\log \left(\frac{f_{2}}{f_{1}}\right)}\right),
$$

where $\hat{T}$ is an approximate time length of the signal $s(t)$ and where Round represents rounding towards nearest integer.

The redesigned exponential swept-sine signal can then be generated using the equations (10) and (22). The parameters which define the input signal $s(t)$ are start and stop frequencies $f_{1}$ and $f_{2}$, and the approximative time length $\tilde{T}$.

\subsection{Time Domain Properties of the Input Signal}

The signal $s(t)$ defined above and satisfying the conditions depicted in Fig. 4 has furthermore the following property. Consider a signal $s_{2}(t)$ with instantaneous frequency equals twice the instantaneous frequency of the signal $s(t)$. The relation between both signals is then

$$
s_{2}(t)=s\left(t+\Delta t_{2}\right)
$$

This condition may be extended for any $m \in \mathbb{N}-\{0\}$, as

$$
s_{m}(t)=s\left(t+\Delta t_{m}\right) \text {. }
$$

In other words all the higher-order harmonic components of the signal $s(t)$ can be expressed as time shifted replica of $s(t)$.

\subsection{Frequency Domain Properties of the Input Signal}

To derive the amplitude spectrum $B_{s}(f)$ of the analytic swept-sine signal $z_{s}(t)$, we use Eq. (8). The second-order derivative of the phase $\varphi_{s}(t)$, is expressed, using Eq. (11) and (13), as

$$
\varphi_{s}^{\prime \prime}(t)=\varphi^{\prime \prime}(t)=\frac{2 \pi f_{1}}{L} \exp \left(\frac{t}{L}\right)=\frac{2 \pi f_{i}(t)}{L} .
$$

Using the Eq. (8) and (25) and the equivalence $f \equiv f_{i}$, the amplitude spectrum, for $f>0$, can be written as

$$
B_{s}(f)=\sqrt{\frac{L}{f}},
$$

as depicted in Fig.5.

\subsection{Inverse Filter}

We consider here the analytic signal of the inverse filter $\tilde{s}(t)$ as

$$
z_{\tilde{s}}(t)=\tilde{s}(t)+j H[\tilde{s}(t)]
$$

and the Fourier transform $Z_{\tilde{s}}(f)$ of the analytic signal $z_{\tilde{s}}(t)$. The inverse filter $\tilde{s}(t)$ convolved with the swept-sine $s(t)$ gives theoretically the Dirac function $\delta(t)$ as well as their analytic signals and thus we can write,

$$
Z_{\tilde{s}}(f)=\frac{1}{Z_{s}(f)}
$$




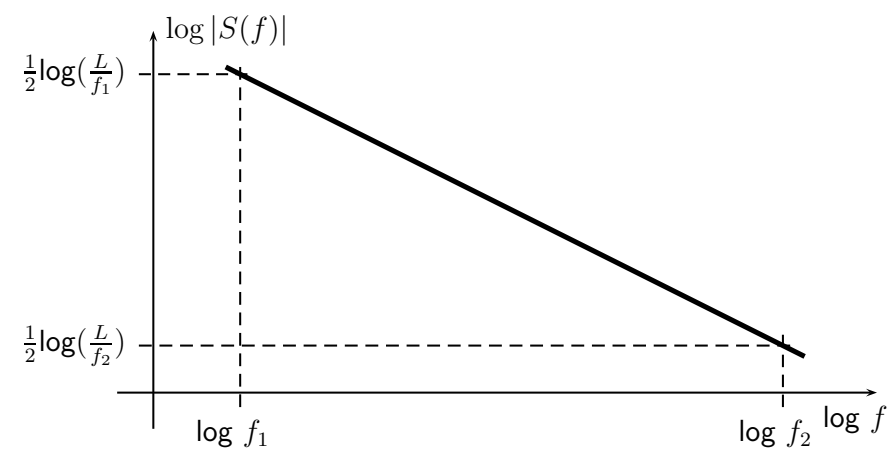

Figure 5. Amplitude of the Fourier Transform of the exponential swept-sine signal.

To express the analytic signal of the inverse filter $z_{\tilde{s}}(t)$, we first derive the relation between magnitudes and phases of both Fourier transforms, as

$$
Z_{\tilde{s}}(f)=\frac{1}{B_{s}(f) e^{j \Psi_{s}(f)}}=\frac{1}{B_{s}(f)} e^{-j \Psi_{s}(f)} .
$$

and thus

$$
\begin{aligned}
& B_{\tilde{s}}(f)=\left|Z_{\tilde{s}}(f)\right|=\frac{1}{B_{s}(f)}, \\
& \Psi_{\tilde{s}}(f)=-\Psi_{s}(f) .
\end{aligned}
$$

For asymptotic signal $z_{s}(t)$, the analytic inverse filter $z_{\tilde{s}}(t)$ is consequently also an asymptotic signal

$$
z_{\tilde{s}}(t)=a_{\tilde{s}}(t) e^{j \tilde{\varphi}_{s}(t)}
$$

Then, we derive the phase $\tilde{\varphi}_{s}(t)$ from the expression of $\tilde{t}_{f}(f)$. Using Eq. (7) and (31), we get

$$
\tilde{t}_{f}(f)=-\frac{1}{2 \pi} \frac{d \Psi_{\tilde{s}}(f)}{d f}=\frac{1}{2 \pi} \frac{d \Psi_{s}(f)}{d f} .
$$

As a consequence, we get

$$
\begin{aligned}
\tilde{t}_{f}(f) & =-t_{f}(f), \\
\tilde{\varphi}_{s}(t) & =\varphi_{s}(-t) .
\end{aligned}
$$

To derive the amplitude $a_{\tilde{s}}(t)$ of the inverse filter, we use the Eq. (8)

$$
B_{\tilde{s}}(f)=\frac{a_{\tilde{s}}\left(\tilde{t}_{f}\right)}{\sqrt{\frac{1}{2 \pi}\left|\tilde{\varphi}_{s}^{\prime \prime}\left(\tilde{t}_{f}\right)\right|}} .
$$

As $\tilde{\varphi}_{s}^{\prime \prime}\left(\tilde{t}_{f}\right)=\varphi_{s}^{\prime \prime}\left(t_{f}\right)$ (from Eq. (34) and (35)), we can substitute from Eq. (25)

$$
B_{\tilde{s}}(f)=\frac{a_{\tilde{s}}\left(\tilde{t}_{f}\right)}{\sqrt{\frac{f}{L}}} .
$$

Now, from Eq. (26),(30) and (37) we can write

$$
a_{\tilde{s}}\left(\tilde{t}_{f}\right)=\frac{f_{i}}{L}
$$


Using Eq. (12), the envelope $a_{\tilde{s}}(t)$ is given by

$$
a_{\tilde{s}}(t)=\frac{f_{1}}{L} \exp \left(-\frac{t}{L}\right)
$$

and the analytic inverse filter is finally expressed as

$$
z_{\tilde{s}}(t)=\frac{f_{1}}{L} \exp \left(-\frac{t}{L}\right) e^{j\left(\varphi_{s}(-t)\right)}
$$

that is in shorten form

$$
z_{\tilde{s}}(t)=\frac{f_{1}}{L} \exp \left(-\frac{t}{L}\right) z_{s}(-t)
$$

The inverse filter $s(t)$ is then

$$
\tilde{s}(t)=\frac{f_{1}}{L} \exp \left(-\frac{t}{L}\right) s(-t)
$$

\section{Principles of the Method of Identification}

In previous section, the input signal $s(t)$ and the inverse filter $\tilde{s}(t)$ have been defined to be used for identification of the NLS under test. This section focuses on the relation between partial frequency responses $H_{m}(f)$ and linear filters $G_{n}(f)$ in frequency domain.

As the impulse responses $h_{m}(t)$ and $g_{n}(t)$, defined as the inverse Fourier transform of $H_{m}(f)$ and $G_{n}(f)$ respectively, are supposed to be real functions, it follows from the hermitian properties of $H_{m}(f)$ and $G_{n}(f)$ that only the half frequency area $f>0$ is considered in the following.

Given the partial frequency responses $H_{m}(f)$ defined by Eq. (2), the frequency response of the linear filters $G_{n}(f)$ of the power series nonlinear model can be derived analytically using the trigonometric power formulas, defined as [23], $\forall l \in \mathbb{N}$

$$
(\sin x)^{2 l+1}=\frac{(-1)^{l}}{4^{l}} \sum_{k=0}^{l}(-1)^{k}\left(\begin{array}{c}
2 l+1 \\
k
\end{array}\right) \sin [(2 l+1-2 k) x],
$$

and $\forall l \in \mathbb{N}-\{0\}$,

$$
\begin{aligned}
(\sin x)^{2 l}= & \frac{(-1)^{l}}{2^{2 l-1}} \sum_{k=0}^{l-1}(-1)^{k}\left(\begin{array}{l}
2 l \\
k
\end{array}\right) \cos [2(l-k) x] \\
& +\frac{1}{2^{2 l}}\left(\begin{array}{c}
2 l \\
l
\end{array}\right) .
\end{aligned}
$$

Regarding the Fourier transform of Eq. (43) and (44) and noting $F T_{p}$ the result of the Fourier transform only for positive frequencies, we can write

$$
F T_{p}\left\{(\sin x)^{2 l+1}\right\}=\frac{(-1)^{l}}{4^{l}} \sum_{k=0}^{l}(-1)^{k}\left(\begin{array}{c}
2 l+1 \\
k
\end{array}\right) F T_{p}\{\sin [(2 l+1-2 k) x]\},
$$

and $\forall l \in \mathbb{N}-\{0\}$,

$$
\begin{aligned}
F T_{p}\left\{(\sin x)^{2 l}\right\}= & j \frac{(-1)^{l}}{2^{2 l-1}} \sum_{k=0}^{l-1}(-1)^{k}\left(\begin{array}{c}
2 l \\
k
\end{array}\right) F T_{p}\{\sin [2(l-k) x]\} \\
& +\frac{1}{2^{2 l}}\left(\begin{array}{c}
2 l \\
l
\end{array}\right) .
\end{aligned}
$$

These formulas give in the Fourier domain the relation between the higher-order harmonic $\sin (l x)$ and the $l$-th power of the harmonic signal $\sin ^{l}(x)$, for $l \in \mathbb{N}$. Considering a harmonic input signal of frequency $f_{0}>0$, the values 
of the frequency responses $H_{m}\left(l f_{0}\right)$ and the values of the frequency responses of the linear filters $G_{n}\left(l f_{0}\right)$ are related in the same way as in (43-44).

The trigonometric formulas in frequency domain can then be rewritten into the matrix form (47), where the matrices A and $\mathbf{B}$ represent the coefficients in Eq. (45) and (46)

$$
\left(\begin{array}{c}
F T_{p}\{\sin x\} \\
F T_{p}\left\{\sin ^{2} x\right\} \\
F T_{p}\left\{\sin ^{3} x\right\} \\
\vdots
\end{array}\right)=\mathbf{A}\left(\begin{array}{c}
F T_{p}\{\sin x\} \\
F T_{p}\{\sin 2 x\} \\
F T_{p}\{\sin 3 x\} \\
\vdots
\end{array}\right)+\mathbf{B} .
$$

The matrix $\mathbf{A}$ is defined, according to Eqs. (45), (46), as

$$
A_{n, m}= \begin{cases}\frac{(-1)^{2 n+\frac{1-m}{2}}}{2^{n-1}}\left(\begin{array}{c}
n-m \\
\frac{n}{2}
\end{array}\right), & \text { for } n \geq \operatorname{mand}(n+m) \text { is even } \\
0, & \text { else. }\end{cases}
$$

The matrix $\mathbf{B}$ is a one-column matrix and represents the constant values of the even power series. These values are only linked to the mean value of the output signal. The relation between the partial frequency response $H_{m}\left(f_{0}\right)$, for $f_{0}>0$ and the linear filters $G_{n}\left(f_{0}\right)$ from the power series nonlinear model is given using the coefficients of the matrix A. Each partial frequency response $H_{m}\left(f_{0}\right)$, or $m$-th harmonic, can be expressed as a sum of $m$-th harmonics of all the $n$-th powers weighted by the linear filters $G_{n}\left(f_{0}\right)$. The coefficients of the $m$-th harmonics of the $n$-th power are $A(n, m)$ and thus

$$
H_{m}\left(f_{0}\right)=\sum_{n=1}^{N} A_{n, m} G_{n}\left(f_{0}\right) .
$$

Lastly, the linear transformation between $H_{m}\left(f_{0}\right)$ and $G_{n}\left(f_{0}\right)$, for $f_{0}>0$ can be generally expressed in matrix form

$$
\left(\begin{array}{c}
G_{1}\left(f_{0}\right) \\
G_{2}\left(f_{0}\right) \\
G_{3}\left(f_{0}\right) \\
\vdots
\end{array}\right)=\left(\mathbf{A}^{T}\right)^{-1}\left(\begin{array}{c}
H_{1}\left(f_{0}\right) \\
H_{2}\left(f_{0}\right) \\
H_{3}\left(f_{0}\right) \\
\vdots
\end{array}\right),
$$

where $\mathbf{A}^{T}$ denotes the transpose of $\mathbf{A}$.

\section{Results}

\subsection{Simulation of a NLS with memory}

In order to illustrate the method, a simulated NLS with memory is identified. This NLS consists of two nonlinear branches with linear and cubic parts, each of them followed by a linear filter (Fig. 6). To simulate real-world

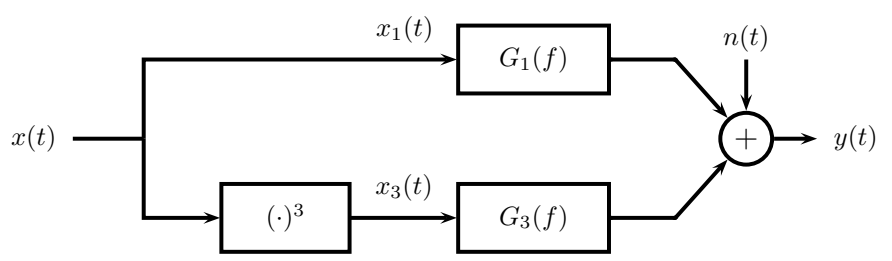

Figure 6. Simulated NLS with memory. 

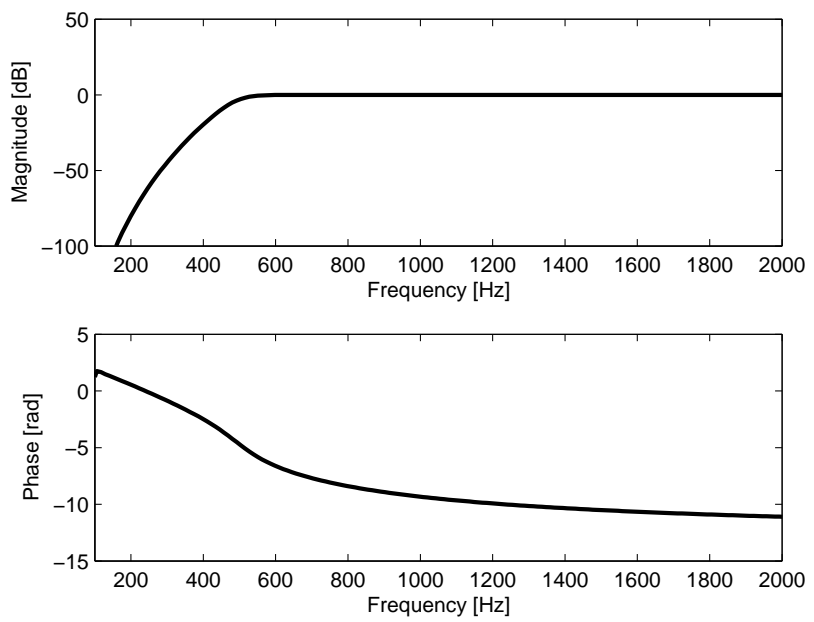

Figure 7. The theoretical frequency response (modulus - above; phase - below) of the filter $G_{1}(f)$ from the linear part of the tested system.
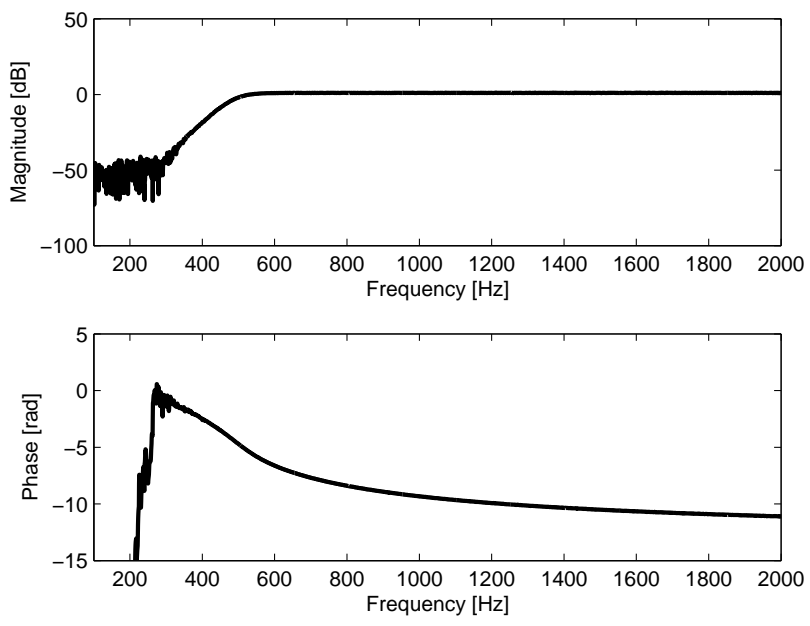

Figure 8. The estimated frequency response (modulus - above; phase - below) of the filter $\hat{G}_{1}(f)$ from the linear part of the tested system.

conditions, a white gaussian noise (WGN) $n(t)$ is added to the output signal. Both filters are digital Butterworth filters. The filter $G_{1}(f)$ used in linear branch is a 10-th order high-pass filter, with cutoff frequency $500 \mathrm{~Hz}$ and the filter $G_{3}(f)$, used in the cubic branch, is a 10-th order low-pass filter, with cutoff frequency $1 \mathrm{kHz}$. The simulation is performed using a sampling frequency $f_{s}=12 \mathrm{kHz}$. The excitation signal used for the identification is a sweptsine signal as defined in Sect.3, with the following parameters: $f_{1}=20 \mathrm{~Hz}, f_{2}=2000 \mathrm{~Hz}, \tilde{T}=5 \mathrm{~s}$. The maximum frequency $f_{2}$ has been chosen in order to avoid any aliasing [24, 25]. The order of the model is set to $N=3$. Once the response of the NLS under test to this excitation signal is known, the nonlinear convolution described in Sect. 4 is performed and the linear filters of the nonlinear model are estimated.

The estimated frequency responses (modulus and phases) of filters $\hat{G}_{1}(f)$ and $\hat{G}_{2}(f)$ are respectively given in Fig. 8 and 10 and compared to the theoretical ones (Fig. 7 and 9) with a SNR equal to 30 dB. In the frequency ranges of the swept-sine input signal $\left(f_{1}=20 \mathrm{~Hz}, f_{2}=2 \mathrm{kHz}\right)$ both estimated filters, the High-Pass filter (500 - $\left.2000 \mathrm{~Hz}\right)$ 

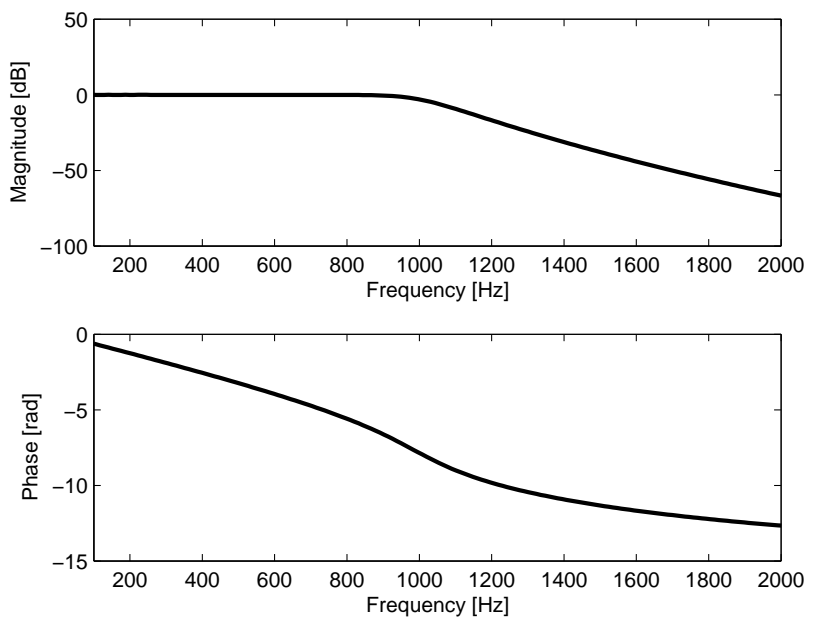

Figure 9. The theoretical frequency response (modulus - above; phase - below) of the filter $G_{3}(f)$ from the nonlinear part of the tested system.
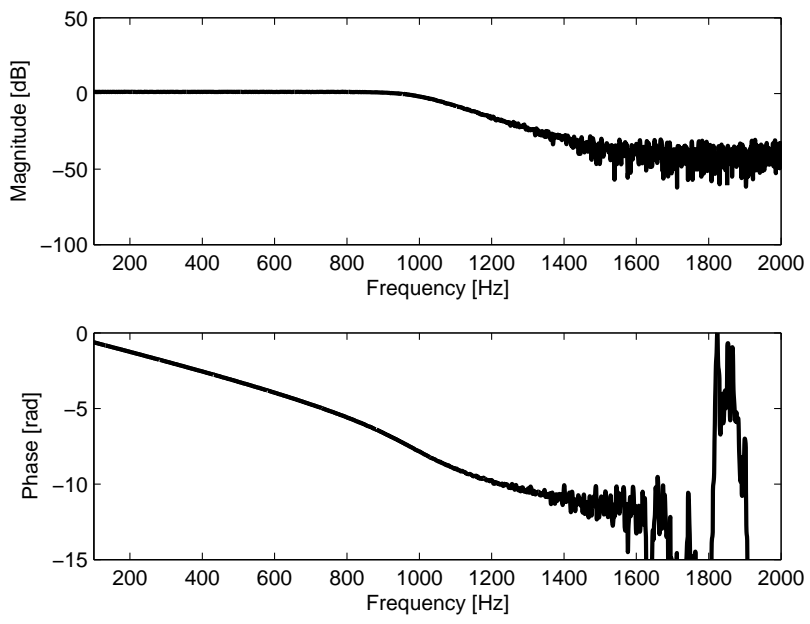

Figure 10. The estimated frequency response (modulus - above; phase - below) of the filter $\hat{G}_{3}(f)$ from the nonlinear part of the tested system.

and the Low-Pass filter $(50-1000 \mathrm{~Hz})$, match in amplitude and phase with the theoretical characteristics.

In order to test the robustness of the identification method, the simulation has also been performed for several levels of additive noise $n(t)$. The mean squared error (MSE) of the estimated filters depends indeed on the SNR. The MSE is calculated as the mean square of the modulus of the complex difference between the estimated and real filters in the frequency range $\left[f_{1}=20 \mathrm{~Hz}, f_{2}=2 \mathrm{kHz}\right]$. No synchronous averaging is performed.

The robustness of the method is illustrated in Figure 11. As expected, the MSE is made of two contributions: the error caused by the additive noise $n(t)$ and the model error [18]. The proposed method estimates the linear filters of the model with an error less than $\simeq 10^{-2}$, even for the lowest simulated SNR. For SNR higher than 50 $\mathrm{dB}$, the MSE remains constant, due to the model error, at a value closed to $\simeq 10^{-6}$. 


\subsection{Identification of a real NLS}

In this section, a real-world NLS is selected for testing the proposed method. The system under test is the limiter part of $d b x$ 266XL Compressor, Limiter, Gate [26]. The limiter is a NLS producing highly distorted output waveforms. The clipping level of the limiter is set to $0.25 \mathrm{~V}$.

The measurements are performed using the swept-sine excitation signal with amplitude $A=1 \mathrm{~V}$, frequencies $f_{1}=10 \mathrm{~Hz}, f_{2}=5 \mathrm{kHz}$ and approximative time duration $\tilde{T}=6 \mathrm{~s}$. The sampling frequency is $f_{s}=96 \mathrm{kHz}$ and the order of the model is $N=8$.

To verify the accuracy of the estimated model, a signal $x(t)$ is generated and used as input signal of both model and real-world limiter. The responses $y_{r}(t)$ of the model and $y(t)$ of the limiter are then compared in both time and frequency domains. Three comparisons, corresponding to three different input signals are performed. As an objective criterion a mean squared residual error between regenerated and measured output waveforms is measured for all the three cases.

Firstly, a sine-wave input signal is generated with frequency $f_{0}=500 \mathrm{~Hz}$ and amplitude $A_{0}=1 \mathrm{~V}$. Both regenerated and real-world system outputs are then compared in Fig. 12. For the sake of clarity, the output of the real limiter in frequency domain is shifted to the right. On one hand, the output of the real-world system consists of numerous higher-order harmonic components. On the other hand, the model is truncated to the 8th order. As a consequence, the regenerated output signal cannot contain more than 8 higher-order harmonic components and then exhibits well-known oscillations, known as Gibbs phenomenon. Nevertheless, the regenerated output signal fits in with mean squared error $\mathrm{MSE}=2.3 \cdot 10^{-4}$.

Secondly, the input signal is a sine-wave with the same frequency $\left(f_{0}=500 \mathrm{~Hz}\right)$, but with a lower amplitude $\left(A_{0}=0.3 \mathrm{~V}\right)$. Both regenerated and real-world system are compared in Fig. 13. It shows that the nonlinear model fits in also well for a lower input signal, even if the 4th odd component is badly estimated, suggesting a possible input-output law depending on the amplitude of the input signal. As for the previous case the mean squared error is $\mathrm{MSE}=2.3 \cdot 10^{-4}$.

Lastly, a sawtooth input signal is used as the input signal and the measured and regenerated output signals are compared. The sawtooth signal is is chosen to exhibit a period of 480 samples, equivalent to frequency $200 \mathrm{~Hz}$, for a data rate $f_{s}=96 \mathrm{kHz}$. The results are depicted in Fig. 14. The regenerated and measured output waveforms are very similar, even if the regenerated one exhibits Gibbs oscillations. The mean squared error between regenerated

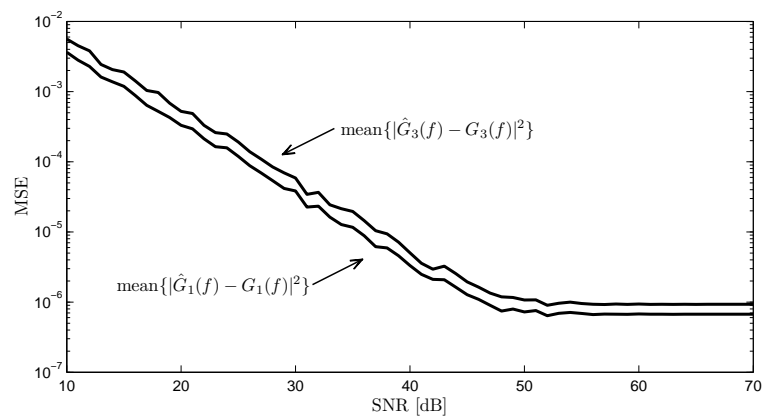

Figure 11. Dependency of MSE of the estimated filters $\hat{G}_{1}(f)$ and $\hat{G}_{3}(f)$ on SNR. 

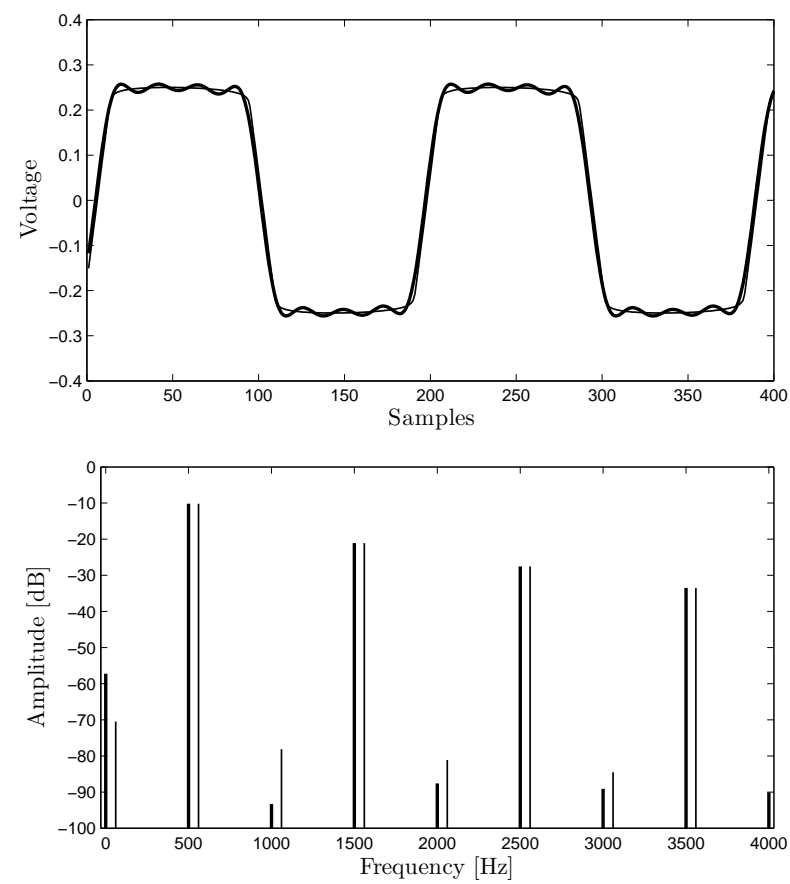

Figure 12. Comparison of the real-world (thin) and regenerated (bold) responses (waveforms - left; spectra - right) to the sine-wave $f=500 \mathrm{~Hz}, A=1 \mathrm{~V}$.
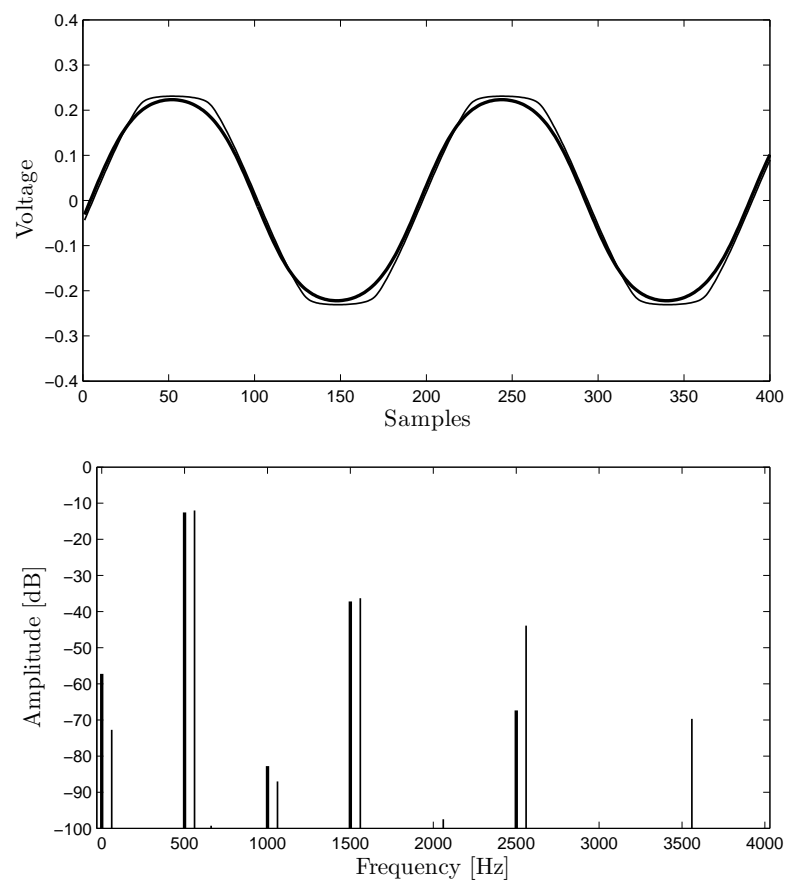

Figure 13. Comparison of the real-world (thin) and regenerated (bold) responses (waveforms - left; spectra - right) to the sine-wave $f=500 \mathrm{~Hz}, A=0.3 \mathrm{~V}$.

and measured output waveforms is $\mathrm{MSE}=6.6 \cdot 10^{-4}$. That confirms that the method is accurate enough for regenerating complex output waveforms. 

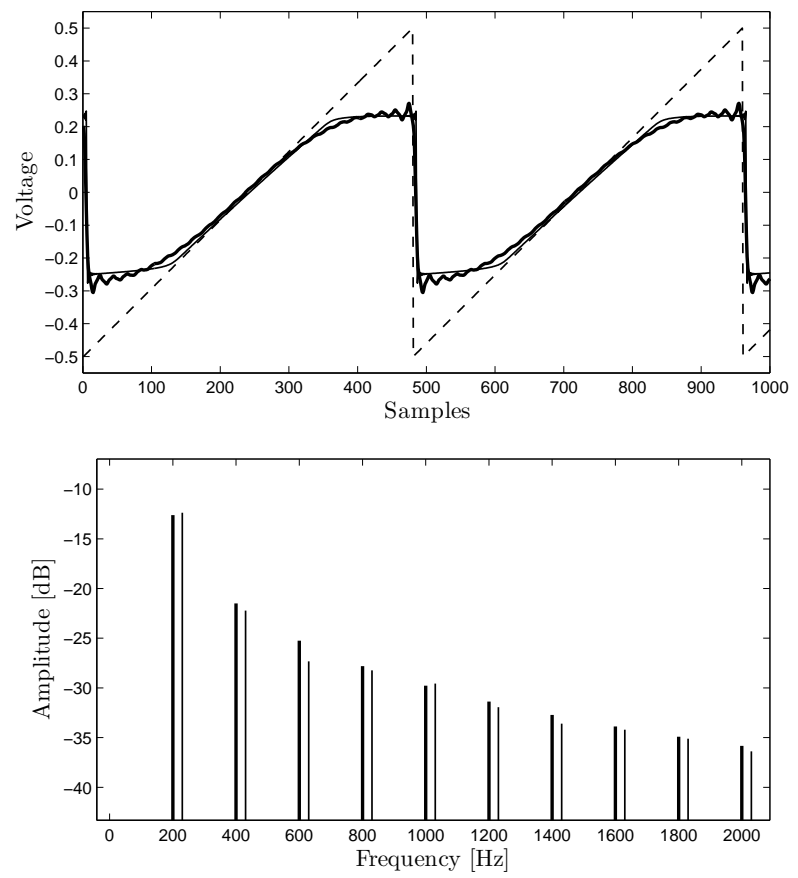

Figure 14. Comparison of the real-world (thin) and regenerated (bold) responses (waveforms - left; spectra - right) to the sawtooth input signal (dashed).

\section{Comparison with Existing Methods}

There is a wide variety of methods in the literature of NL system identification. On one hand, methods based on Wiener, Hammerstein, Multiple Input Single Output (MISO), or block-oriented models may be considered as methods based on dedicated models [18]. On the other hand, methods based on neural network, extended Kalman filtering, particle filtering are not based on specific models and are usually regarded as universal approximators [18].

It is difficult to make a detailed comparison with all existing methods because the models and the methodologies associated to each method are too different. As the identification method proposed in this paper is based on a polynomial structure model, the comparative study of this section concerns methods based on similar models: Hammerstein model and Wiener model. The global advantages of the proposed method is summarized at the end of this section.

The criterion used to perform the comparison is the mean squared error (MSE) between the regenerated output $y_{r}(t)$ and the output $y(t)$ of the system under test. Two systems are identified in order to perform the comparison: the real audio limiter previously identified in section 5.2 and also an equivalent simulated limiter (zero-memory system). The comparison is carried out for the three signals used in section 5.2. All the methods are based on 8th degree polynomial model. The Wiener and Hammerstein methods are implemented using the MAtLAB - System Identification Toolbox. Table 1 summarizes the MSE obtained for each method, for both simulated and real-world system identifications.

As seen in Table 1, for simulated data, the Swept-Sine method leads to MSE of the same order of magnitude of the ones linked to other methods. This can be explained by the fact that all tested methods use the same polynomial structure. Moreover, the results for real-word system identification clearly shows that the Swept-Sine method performs better for all the tested cases. 
Table 1. Mean squared errors.

\begin{tabular}{lcc} 
& simulation & real-world \\
\hline \multicolumn{3}{c}{ sine wave with $A=1 \mathrm{~V}$} \\
NL convolution & 0.00011 & 0.00023 \\
Hammerstein & 0.00014 & 0.0014 \\
Wiener & 0.00014 & 0.0015 \\
\hline \multicolumn{3}{c}{ sine wave with $A=0.3 \mathrm{~V}$} \\
NL convolution & 0.00026 & 0.00023 \\
Hammerstein & 0.00018 & 0.0032 \\
Wiener & 0.00018 & 0.0041 \\
\hline \multicolumn{3}{c}{ sawtooth signal with $A=0.5 \mathrm{~V}$} \\
NL convolution & 0.00054 & 0.00066 \\
Hammerstein & 0.00016 & 0.0025 \\
Wiener & 0.00017 & 0.0031 \\
\hline
\end{tabular}

\section{Conclusion}

In this paper, a method for identification of nonlinear systems is presented. The result of the identification is a nonlinear model consisting of nonlinear branches, each branch being in the form of $n$-th power in series with a linear filter, equivalent to generalized polynomial Hammerstein model. The method allows in particular to regenerate an output signal corresponding to any given input signal and to compare this regenerated output signal to the realworld output, in order to validate the accuracy of the model.

The method has been applied to a real-world nonlinear system (NLS) (audio limiter). The nonlinear model has been tested for different input signals, two sine-wave signals with different level and a sawtooth signal and compared with other methods.

On one hand, one of the characteristics of the method is the necessity of properly designed excitation signal. For that reason the system under test cannot be excited from a simple signal generator, but from a personal computer with an audio card, or from signal generator with memory, in which the properly designed signal is recorder.

On the other hand, the robustness of the method has been proved regarding noise characteristics (Fig. 11) and through a real measurement test (Table 1), as also presented in [16, 17]. It has also to be noted that the method has a low calculation time cost. Compared to the methods that are not based on a specific model (Extended Kalman Filtering, Particle Filtering, Neural Networks), the proposed method is straightforward with no special algorithms and thus easy to be implemented. In addition, the method has no need of any knowledge of the system under test.

\section{Acknowledgment}

This work has been supported by Ministry of Education in Czech Republic under research program "Research in the Area of the Prospective Information and Navigation Technologies" No. MSM6840770014 and by the French Embassy in Prague within the framework of the program "Cotutelle de thèse". 


\section{References}

[1] T. Kailath, Linear Systems. NJ, Prentice-Hall: Englewood Cliffs, 1980.

[2] B. P. Lathi, Signal Processing and Linear Systems. Oxford, UK: Oxford University Press, 2000.

[3] M. Schetzen, The Volterra and Wiener theories of nonlinear systems. New York: John Wiley \& Sons, 1980.

[4] O. Nelles, Nonlinear System Identification: From Classical Approaches to Neural Networks and Fuzzy Models. Berlin: Springer, 2001.

[5] J. S. Bendat, Nonlinear System Techniques and Applications. New York, USA: John Wiley \& Sons, 1998.

[6] F. Thouverez and L. Jezequel, "Identification of NARMAX models on a modal base," J. Sound. Vib., vol. 189, no. 2, pp. 193-213, 1996.

[7] H. E. Liao and W. S. Chen, "Determination of nonlinear delay elements within NARMA models using dispersion functions," IEEE Trans. Instrum. Meas., vol. 46, no. 4, pp. 868-872, 1997.

[8] Y. W. Chen et al., "Blind nonlinear system identification based on a constrained hybrid genetic algorithm," IEEE Trans. Instrum. Meas., vol. 52, no. 3, pp. 898-902, 2003.

[9] H. Sorenson, Kalman Filtering: Theory and Application. Montvale, NJ: IEEE Press, 1985.

[10] O. Cappé et al., "An overview of existing methods and recent advances in sequential monte carlo," IEEE Proc. Signal Proc., vol. 95, no. 5, pp. 899-924, 2007.

[11] P. Crama and J. Schoukens, "Initial estimates of wiener and hammerstein systems using multisine excitation," IEEE Trans. Instrum. Meas., vol. 50, no. 6, pp. 1791-1795, 2001.

[12] M. Solomou et al., "Frequency domain analysis of nonlinear systems driven by multiharmonic signals," IEEE Trans. Instrum. Meas., vol. 53, no. 2, pp. 243-250, 2004.

[13] E. Ceri and D. Rees, "Nonlinear distortions and multisine signals-part I: Measuring the best linear approximation," IEEE Trans. Instrum. Meas., vol. 49, no. 3, pp. 602-609, 2000.

[14] A. H. Tan and K. Godfrey, "The generation of binary and near-binary pseudorandom signals: an overview," IEEE Trans. Instrum. Meas., vol. 51, no. 4, pp. 583-588, 2002.

[15] R. Haber and L. Keviczky, Nonlinear system identification: input/output modeling approach, Vols. 1 and 2. Dordrecht: Kluwer Academic Publishers, 1999.

[16] A. Farina, "Simultaneous measurement of impulse response and distortion with a swept-sine technique," in AES 108th convention, Paris, Feb. 2000.

[17] E. Armelloni et al., "Non-linear convolution: A new approach for the auralization of distorting systems," in AES 110th convention, Amsterdam, May 2001.

[18] J. Schoukens et al., "Identification of nonlinear and linear systems, similarities, differences, challenges," in 14th IFAC Symposium on System Identification, Newcastle, Australia, 2006, pp. 122-124.

[19] L. Cohen, Time-Frequency Analysis. NJ, Prentice-Hall: Englewood Cliffs, 1995.

[20] P. Flandrin, Time-frequency/time-scale analysis. San Diego, CA: Academic Press Inc., 1999. 
[21] L. Cohen, "Instantaneous frequency and group delay of a filtered signal," Journal of the Franklin Institute, vol. 337, no. 4, pp. 329-346, 2000.

[22] T. Kite, "Measurement of audio equipment with log-swept sine chirps," in AES 117th convention, San Francisco, CA, USA, Oct. 2004.

[23] W. H. Beyer, Standard Mathematical Tables. Boca Raton, FL, USA: CRC Press, Inc., 1987.

[24] Y. M. Zhu, "Generalized sampling theorem," IEEE Trans. Circuits Syst. II, vol. 39, pp. 587-588, 1992.

[25] J. Tsimbinos and K. V. Lever, "Input Nyquist sampling suffices to identify and compensate nonlinear systems," IEEE Trans. Signal Process., vol. 46, no. 10, pp. 2833-2837, 1998.

[26] "Dbx : Profesional products, dbx 266xl compressor-gate," WWW page, 2007. [Online]. Available: http://www.dbxpro.com/266XL/266XL.php 